90 per cent of all near-Earth asteroids larger than 300 metres across.

The panel's highest-priority 'moderate' mission is not the most expensive on the list, but many astronomers consider the proposed $\$ 50$ million Telescope System Instrumentation Program to be vital to the health of their field. By funding new instruments for private telescopes such as the Keck, which is owned by California universities, the federal government will be buying a certain amount of access for all US astronomers.

Other moderate initiatives include the Gamma-ray Large Area Space Telescope (GLAST), which will succeed the Compton Gamma Ray Observatory; a pioneering gravity-wave telescope in space called LISA (for Laser Interferometer Space Antenna); an Advanced Solar Telescope; and - a rarity for astronomy - an instrument to be placed on the International Space Station, known as the Energetic X-ray Imaging Survey Telescope (EXIST).

The top 'small' initiative identified in the report is the National Virtual Observatory (NVO), a programme to compile astronomical data gathered in different wavelengths into a single large database accessible to scientists and the public. John Bahcall, an astrophysicist with the Princeton Institute for Advanced Study who led the last decadal review in 1991, likens the NVO to a hot "growth stock" on Wall Street. "Many of the most important discoveries will come from mining this database," he says.

The overall programme proposed by the McKee-Taylor panel would cost $\$ 4.7$ billion up to $2010-20$ per cent more than the wish list offered by Bahcall's committee ten years
Prioritized equipment initiatives and estimated federal costs, 2000-10

Initiative Cost $(\$$ million $)$

\section{Major initiatives}

Next Generation Space Telescope (NGST) $\quad 1,000$

Giant Segmented Mirror Telescope (GSMT) 350

Constellation-X Observatory $\quad 800$

Expanded Very Large Array (EVLA) $\quad 140$

Large-Aperture Synoptic Survey Telescope (LSST) 170

Terrestrial Planet Finder (TPF)

200

Single-Aperture Far InfraRed (SAFIR) Observatory 100

Moderate initiatives

Telescope System Instrumentation Program (TSIP) 50

Gamma-ray Large Area Space Telescope (GLAST) 300

Laser Interferometer Space Antenna (LISA) 250

Advanced Solar Telescope (AST) $\quad 60$

Square Kilometre Array (SKA) technology

development

Solar Dynamics Observer (SDO) 22

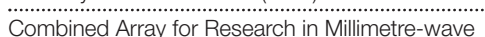

Astronomy (CARMA)

Energetic X-ray Imaging Survey Telescope (EXIST) 150

Very Energetic Radiation Imaging Telescope Array

System (VERITAS)

Advanced Radio Interferometry between Space

and Earth (ARISE)

35

Frequency Agile Solar Radiotelescope (FASR) 26

South Pole Submillimetre Telescope (SPST) $\quad 50$

Small initiatives

National Virtual Observatory (NVO)

Other small initiatives

Total for all initiatives

246

ago. Early in its deliberations, the committee asked NASA and the National Science Foundation, the two funding agencies responsible for most US astronomy, how fiscally conservative it should be, and were told "not to restrain ourselves", says Taylor.
If past decadal reviews are any guide, the new report should be well received on Capitol Hill and in the White House budget office. All four major initiatives proposed by Bahcall's committee in 1991 have since been approved, as have nine out of 11 moderatesize projects. Indeed, the astronomy surveys are almost unique in their influence on Washington decision-makers.

William Wulf, president of the National Academy of Engineering, believes the reason is that they "are not typical please-sendmoney reports — they represent some tough decisions".

Indeed, says Bahcall, the exercise is a "heart-wrenching" process in which astronomers "have to turn down proposals that were a decade in the making".

The committee also tried to consider input from the wider astronomical community, not just the élite, according to Lee Anne Willson, an astronomer at Iowa State University who chairs the astronomy section of the American Association for the Advancement of Science.

She set up a web page for rank-and-file astronomers to provide comments to the survey committee, and says that the comments were read. The committee's final decisions were also thoroughly vetted - after the sub-panels and panels did their work, another 23 readers weighed in with opinions as part of the National Research Council's review process.

The report probably comes as close to a consensus as it could, given the time it took to produce, says Willson. "Astronomy should be proud of itself."

http://books.nap.edu/catalog/9839.html

\title{
Britain asks to join European Southern Observatory
}

\section{David Dickson, London \& Alison Abbott, Munich}

Keen to see the creation of a single European body with the clout to raise the funds needed for future large telescopes, Britain is this week opening negotiations about possible membership of the European Southern Observatory (ESO).

Based in Munich, the eight-member ESO is responsible for the construction and operation of a number of telescopes in the Southern Hemisphere, notably the Very Large Telescope (VLT) currently being built on Mount Paranal in Chile.

Britain declined to join the organization when it was set up in 1962, claiming that it could not afford to do so. But Ian Halliday, the chief executive of the Particle Physics and Astronomy Research Council (PPARC), said last week that are now several reasons for becoming a member.

As well as giving British astronomers access to the VLT, membership of the ESO

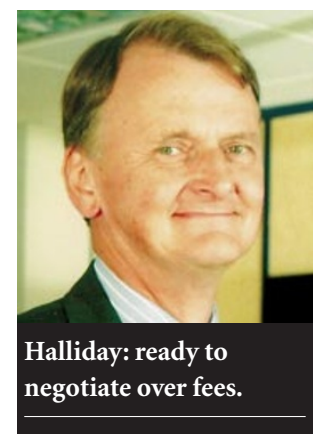

would allow the PPARC to become directly involved in the planning of major new international facilities, such as the proposed optical telescope known as the Overwhelmingly Large Telescope (OWL).

"In 10 years' time, if we want to build this type of facility, we need to be part of an organization such as ESO," says Halliday. "We will not be able to afford to build it ourselves."

Many members of the ESO, including its director Catherine Cesarsky, are happy for Britain to join. Franco Pacini, a member of the ESO council and director of the Arcetri
Astrophysics Observatory in Florence, says: "No-one would deny how important it will be for ESO to have Britain as a member. It is a pity that [Britain] did not join long ago."

But agreeing on the terms of entry will not be easy. The treaty setting up the ESO stipulates that new members must pay an entry fee as a contribution to the capital costs of the facilities they will be able to use.

In Britain's case, the entry fee would be $\mathfrak{E} 55$ million (US\$81.8 million). But Halliday says that, given the pressures on the UK science budget, paying such a sum is out of the question. "If they are insistent about us paying our full whack, we will not be able to get into ESO," he says.

One ESO official says: "It is an unfortunate moment [for Britain to ask for special treatment] because ESO has just made a huge investment in the VLT, and member states may not be too happy about 
Britain joining without making a retrospective contribution."

Cesarsky says that the ESO will enter negotiations with a flexible outlook. An offer in kind - for example, giving astronomers from ESO member states access to British telescopes at Paranal would be considered seriously. But to suggest that the ESO would reduce the total value of the entry fee is, she says, "pure dreaming".

Even if the question of the entry fee is settled, there is also the issue of the annual payments, which would currently amount to $£ 12$ million a year. Without a significant increase in government funding to cover this sum, there would have to be cuts in other parts of the PPARC's budget.

Some of the money could be found, for example, by closing some of Britain's existing telescopes on La Palma in the Canary Islands. This prospect is worrying astronomers working on the facilities. There is also concern from particle physicists that covering a commitment to the ESO could be achieved by taking money away from accelerator research.

But the importance to British astronomers of joining the ESO was underlined in a report, Unveiling the Universe, published by the PPARC last week. It lists joining the $\mathrm{ESO}$ as high among its priorities. Reflecting the priorities of the PPARC's astronomy panel, the report suggests that the research council should seek an extra $£ 39$ million from the government over two years.

Halliday is not only confident of success in his negotiations with the ESO, but also optimistic that his efforts to build closer European collaboration will be received sympathetically in Whitehall. "ESO is currently not a well funded organization," he says. "They probably have more telescopes than they can afford. Somewhere in there is a potential for negotiation."

However, Cesarsky says that the ESO is not so strapped for cash that it needs to compromise to suit Britain's pocket.

"Britain knows what the conditions are for joining," she says.

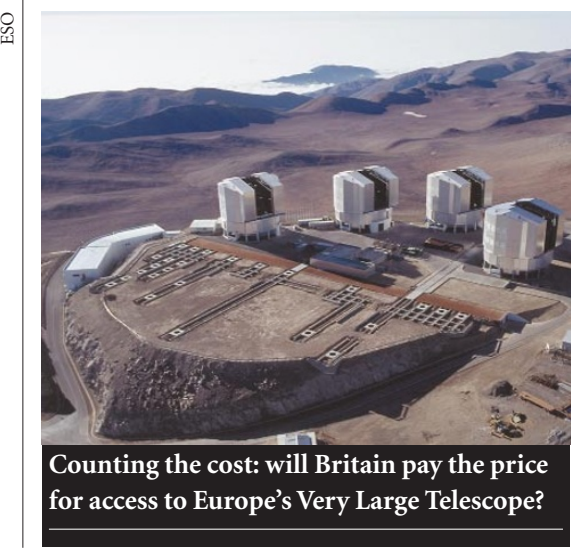

\section{Mathematicians chase the seven million-dollar proofs}

\section{David Dickson}

Mathematics might be the queen of the sciences, but as far as international prizes go, its omission from the list of Nobel disciplines has made it a poor relation.

All that, however, is about to change. The recently established Clay Mathematics Institute (CMI) in Cambridge, Massachusetts, was due to announce this week that it is offering \$1 million for the proof of each of seven classical mathematical problems.

Among them is the Riemann hypothesis concerning the distribution of prime numbers - widely considered the most important problem in number theory - and the Navier-Stokes equations familiar to anyone who has studied fluid dynamics.

Also listed is the ' $\mathrm{P}$ vs NP' (polynomial versus non-deterministic polynomial) problem. This concerns the relationship between the difficulty of solving a problem and of checking solutions, and bears directly on the theoretical possibility of being able to break computer codes (see Nature 400, 115-116; 1999).

"We wanted to make a statement to the general public that maths is important," says Arthur Jaffé, professor of mathematical physics at Harvard University and a member of the CMI's Science Advisory Board. "We believe that the future of the world is going to be dependent on science, and that mathematics is at the centre."

Jaffé was largely responsible for persuading businessman Landon T. Clay, 18 months ago, to set up a non-profit foundation dedicated to increasing and disseminating mathematical knowledge. The CMI already funds a range of activities, from research fellowships to workshops and summer schools. The institute now hopes that its decision to offer a series of prizes will recreate the type of public fervour that accompanied the solution of Fermat's last theorem in 1993 by the British-born mathematician Andrew Wiles.

"We wanted to do something original," says Alain Connes, professor of analysis and geometry at the Collège de France in Paris, which hosted the meeting at which the prizes were to be announced. Like Wiles, Connes is a member of the CMI's four-member Science Advisory Board.

It is almost a century since German mathematician David Hilbert set out 23 key unresolved problems that he predicted would set the agenda for mathematics in the new century, during a famous lecture in Paris, on 8 August 1900. His prediction was accurate. The problems he set out dominated much of mathematics in the past 100 years, and all but three have been resolved. (The solution of

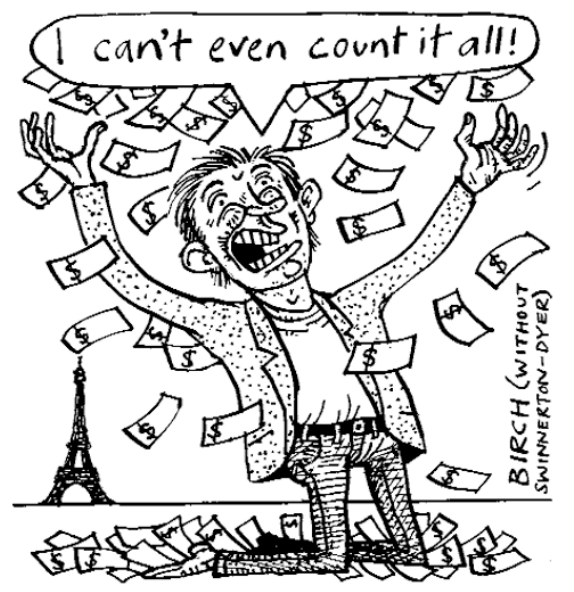

the Riemann hypothesis is one of the three outstanding problems from Hilbert's list.)

In contrast to Hilbert's speech - and reflecting concern in some quarters that the CMI might be attempting a similar task those responsible for the prize emphasize that they have no desire to determine the direction in which mathematics moves forward.

Rather, they are trying to recreate excitement about the activity of mathematics, both in the general public and among school students. "Sometimes people have the wrong idea of maths and think that it will be overtaken by computers," says Connes. "The seven problems, each selected by top specialists in their respective fields, are totally inaccessible to computers."

The competition has strict rules for determining whether a problem has actually been solved. In particular, a proposed solution must be published in a refereed journal "of worldwide repute", and must have gained "general acceptance" in the mathematics community within two years of publication. Also, to ensure that the lure of a reward does not encourage mathematicians to lock themselves away and work on the problems in private, prior work will be taken into account in awarding a prize.

There is no time limit to the competition. "We know that they are difficult, because people have been working on them for a long time," says Jaffé. But he also expresses optimism: "I would hope to live to see each of these solved."

The $\$ 1$ million problems are:

- The P vs NP problem

- The Riemann hypothesis

- The Poincaré conjecture

- The Hodge conjecture

- The Birch and Swinnerton-Dyer conjecture

- Navier-Stokes equations

- Yang-Mills theory

http://www.claymath.org 